\title{
Philosophie politique des multitudes. Mineure. Créoles, a cura di Jean-Yves Mondon e Laurent Bove
}

\section{Alessandro Corio}

\section{(2) OpenEdition}

12 Journals

\section{Edizione digitale}

URL: https://journals.openedition.org/studifrancesi/26911

DOI: 10.4000/studifrancesi.26911

ISSN: 2421-5856

\section{Editore}

Rosenberg \& Sellier

\section{Edizione cartacea}

Data di pubblicazione: 1 avril 2007

Paginazione: 219-221

ISSN: 0039-2944

\section{Notizia bibliografica digitale}

Alessandro Corio, «Philosophie politique des multitudes. Mineure. Créoles, a cura di Jean-Yves Mondon e Laurent Bove», Studi Francesi [Online], 151 (LI | I) | 2007, online dal 30 novembre 2015, consultato il 23 novembre 2021. URL: http://journals.openedition.org/studifrancesi/26911 ; DOI: https://doi.org/ 10.4000/studifrancesi.26911

Questo documento è stato generato automaticamente il 23 novembre 2021.

\section{(c)}

Studi Francesi è distribuita con Licenza Creative Commons Attribuzione - Non commerciale - Non opere derivate 4.0 Internazionale. 


\title{
Philosophie politique des multitudes. Mineure. Créoles, a cura di Jean-Yves Mondon e Laurent Bove
}

\author{
Alessandro Corio
}

\section{NOTIZIA}

«Multitudes», Philosophie politique des multitudes. Mineure. Créoles, a cura di Jean-Yves Mondon e, n. 22, septembre 2005, pp. 167-221.

Questo numero della rivista francese «Multitudes» presenta un'interessante sezione "minore" dedicata alle possibili declinazioni intellettuali, teoriche, politiche e letterarie del "creolo", inteso nelle sue accezioni storico antropologiche e linguistiche, ma anche, e soprattutto, nelle interessanti prospettive di dibattito politico che, negli ultimi due decenni, si sono aperte a partire dalla produzione letteraria delle Antille e che, come è noto, attraverso i fortunati concetti di "créolité" e di "créolisation", introdotti da Glissant e dai suoi "discepoli" Confiant, Bernabé e Chamoiseau col famoso elogiomanifesto del 1991, hanno fornito alcuni stimoli essenziali alla riflessione sulle dinamiche delle società cosiddette "multiculturali" e sui processi antropologici in atto nella "globalizzazione". Quella del "creolo" risulta quindi, ben oltre la specifica collocazione storico-geografica di matrice caraibica, una categoria poetica, antropologica e politica al tempo stesso, chiamata a giocare un ruolo sempre più importante e controverso nelle configurazioni politiche del nostro presente. I cinque articoli presenti in questa sezione della rivista affrontano, da punti di vista differenti ed in parte conflittuali, tanto la genesi storico-antropologica e linguistica delle società creole, quanto le aperture, le prospettive e le contraddizioni che le teorie della "creolizzazione" hanno proposto negli ultimi anni. La rivista ha il merito di trasporre questi concetti dall'ambito disciplinare più ristretto e "famigliare" delle letterature francofone ad un terreno più aspro e conflittuale, e di respiro più ampio, che ne mette 
alla prova la validità e la traducibilità nell'arena teorica e politica segnata dai cambiamenti e dai conflitti degli ultimi anni.

2 L'articolo di Jean-Yves Mondon, intitolato La parole du créole qui ne se dit pas «créole» en créole (pp. 167-178), ha il merito di rendere conto della complessità e delle stratificazioni culturali e sociali sottese all'uso del termine "creolo", a partire da un contesto specifico, quello dei "Mascareignes", dove l'impiego stesso della parola non ha un significato puramente denotativo-descrittivo, ma rende conto del carattere e del significato morale di un "luogo". " "Créole" désigne en mauricien les descendants d'esclaves africains là ou il survivent. On peut employer le mot comme une injure, comme une manière de tracer une frontière, pour donner un exemple de ce qu'il ne faut pas faire et qu'il ne faut pas surtout encourager» (p. 169). Esiste quindi un marcato conflitto sociale, sottolineato dal fatto che risulti indelicato e privo di pertinenza l'utilizzo del termine al di fuori della sfera in cui il lessico sociale condiviso delimita il risultato umano dell'economia di piantagione. Questo uso specifico è la conseguenza e l'espressione della formidabile "razializzazione" che è tutt'oggi alla base della società mascaregna, in cui degli elementi fisici superficiali, come le sfumature di colore della pelle, la forma del naso o il modo di pronunciare determinate parole sono immediatamente vissute come significanti sociali in grado di mobilitare desideri di solidarietà o di esclusione e differenti attitudini nei confronti delle risultanti del "meticciato". La stessa "educazione creola", intesa come sviluppo delle proprie attitudini sociali e culturali all'interno di questa lingua, implica, secondo l'autore, un vero e proprio affinamento sensoriale atto al riconoscimento, a livello dei corpi, della parola, dei gesti, di elementi caratterizzanti le differenti forme dell'incontro "interrazziale". Le persone diventano così un prolungamento dei loro corpi, su cui si può "misurare" con un colpo d'occhio la quantità di "creolo" che li contraddistingue e valutarne così i "mondi possibili" di cui sono espressione e le modalità di desiderio e di relazione che possono generarsi dall'incontro. I contesti originati dalle forme di meticciato risultano estremamente diversificati, così come il valore sociale che gli viene attribuito; di conseguenza, se esistono spazi in cui il termine "créolité" può giocare un ruolo federativo ed alternativo ad un'identità razziale intollerante, esistono altri spazi geografici e politici dove sarebbe perlomeno sorprendente formulare un elogio della "créolité". Lo spazio in cui il termine "creolo" produce senso risulta quindi estremamente diversificato e pertanto non riconducibile a categorie "positive" quali quelle elaborate, negli ultimi anni, dai teorici della "créolité" intesa come aggregato interazionale positivo o della "créolisation imprévisible", che produce nuovi modi di dire, scrivere o pensare. Questi termini corrono quindi il rischio di astrarre in una sfera di pura rappresentazione concettuale quelle che sono le tensioni "carnali" che si generano nelle varie forme di "matrimonio interrazziale" presenti nelle società creole, le quali generano a loro volta forme differenti di esclusione e rifiuto oppure di alleanza e di cooperazione. Quello che manca, secondo Mondon, alle teorie della "créolité/ créolisation" è proprio il fatto di considerare le sfere del corpo e della sessualità come centrali in questi processi, per cui si tende a superare spontaneamente ed in modo ritenuto quasi "miracoloso" attraverso il meticciato - che non rappresenta nulla di "semplice" o "prevedibile" e che implica significati culturali più ampi del "contatto di culture" - quelle forme sociali di interdizione, di separazione e di assenza di contatto fisico e quindi di trasgressione che il termine "creolo", al contrario dei suoi usi "intellettuali", tende socialmente a definire. Si tratta pertanto di uno spazio che ha abbandonato, ormai da tempo, qualsiasi interesse per un'origine, una genesi o una 
genealogia, anche in negativo, del proprio divenire presente. Al contrario, quello creolo è «un espace clivé de plusieurs façons, avec ses fractures et ses frontières, ouvertes ou tracées à même le corps, et avec toutes les transgressions, les fuites, que l'on aimerait pouvoir continuer à couronner du nom de «marronnage», si on veut bien retenir de cette vieille pratique créole le fait de se soustraire aux codes de travail qui plient les corps à des productions qui leur sont parfaitement étrangères et mortifères» (p. 172). Lo "spazio creolo" consiste perciò in uno spazio del "frammento", in cui tutto ciò che precede si è dissolto e dove soltanto il desiderio illetterato, lontano dalle istituzioni e dai loro linguaggi, può produrre l'idioma e il senso che gli è proprio.

3 L'articolo di Raphaël Confiant, dal titolo La créolité contre l'enfermement identitaire (pp. 179-185), ripropone, senza grosse novità, i contenuti elaborati, ormai da quindici anni, dai teorici della créolité, che ne fanno un principio di resistenza ad ogni forma di chiusura identitaria. Senza negare la giustezza e la validità di gran parte di questi argomenti, si renderebbe ormai necessaria, a nostro parere, un'ulteriore evoluzione teorica che prenda in considerazione le critiche mosse da molti, tra cui il loro "padre spirituale", Édouard Glissant, a questa nuova forma ideologica, misurandone pertanto lo scarto nei confronti di un contesto socio-culturale ed economico, quello dei D.O.M., che sembra spingersi sempre più lontano da queste prospettive ideali. Pertanto, anche alla luce di queste critiche, non può non sembrarci teoricamente ingenuo e superato $\mathrm{e}$ politicamente pericoloso, oggi, un punto di vista che parte dalla contrapposizione rigida tra le "false" ideologie identitarie della "Blanchitude", "Négritude" o "Indianité" e l'indiscutibile verità della "réalité créole", che le prime avrebbero mascherato o rimosso, senza peraltro fare grosse distinzioni tra forme ideologiche o discorsive di tipo coloniale e assimilazionista e movimenti di resistenza e di opposizione all'imperialismo occidentale.

4 Di ben altro calibro l'intervento di Alexandre Alaric, Le migrant nu. "Le déporté sur des frontières" (pp. 187-201), il quale annoda abilmente e con raro spessore filosofico e linguistico alcune fila del pensiero complesso ed innovatore di Édouard Glissant, rintracciando così nella sua "antropologia poetica" gli elementi di una poetica della migrazione mondiale e della moltitudine, che possa entrare fecondamente in relazione con le complesse ed ambivalenti dinamiche antropologiche postmoderne, troppo spesso compresse tra allarmismi securitari e xenofobi e forme di cittadinanza ancora saldamente ancorate all'idea dello Stato-nazione. L'esperienza del migrante, trattenuto o deportato attraverso delle frontiere, rappresenta senz'altro un'esperienza drammatica e caotica di rottura e di passaggio all'interno dell'ordine del capitalismo globale e comporta, dal punto di vista umano ed esperienziale, un vero e proprio tentativo di una "nuova nascita", che trova pertanto un antenato nell'esperienza di quello che Glissant definisce "le migrant nu", ossia lo schiavo deportato nelle piantagioni. La "créolisation des mondes", di cui parla Glissant, non è quindi altro che questa continua esperienza della rottura e della difficile "venuta al mondo" all'interno dei meccanismi del nuovo ordine produttivo mondiale: "Avènement d'une forme inédite de la genèse de sens dans des communautés, de ce qui est en jeu dans la possibilité même du parler, dans cette double incertitude de la possibilité de l'échange avec les autres et avec le paysages de l'arrivée, dans la décentration du monde, de la finitude et de la solitude" (p. 189). Rintracciare quest'esperienza nell'articolato percorso teorico e poetico di Glissant significa innanzitutto imbattersi in un pensiero della "traccia" intesa come concetto aperto, non totalizzante, e dimensione cognitiva del rapporto al mondo che, oltre la sofferenza del "grido" e del suo "inaudible écho" e 
al di là del tempo lineare della Storia e del Discorso, comporta un'accumulazione primitiva di soggettività. Quest'ultima si esprime e trova spazio nella relazione fondante con l'altro e in un "Noi" polifonico che non è frutto di una genesi, ma che, proprio per questo, può essere in grado di generare delle strategie sociali di resistenza all'ordine dominante. Ripercorrendo alcune "tappe" fondamentali di quest'autore centrale della nostra epoca, dalla poetica dell'erranza e della Relazione all'identità rizomatica, dalle strutture romanzesche polifoniche ed imprevedibili al rapporto "profetico" della comunità col proprio passato, Alaric ritrova in Glissant l'espressione di un "nuovo modo di essere al mondo" come scoperta della propria voce in presenza di tutti i popoli, i luoghi e le lingue del mondo, esperienza enigmatica e paradossale della presenza del tutto nella parte, che è all'origine della Parola e che qualifica come poche altre la condizione delle moltitudini, migranti e non, che affollano oggi il nostro pianeta.

5 Nell'intervento di Yves Citton, Créolectures et politiques membraniques (pp. 203-211), troviamo forse l'evoluzione ontologica e politica più interessante ed originale della teoria della creolizzazione di Glissant. L'autore rintraccia l'elemento qualificante della categoria di "creolo" nello spostamento ("déplacement"), non solo di genti e di popoli, ma di lingue, accenti, pratiche, significati ed immaginari. Se ne deduce che l'atto stesso della lettura, come è stato sottolineato a più riprese dai principali teorici della semiotica e dell'ermeneutica, da Gadamer a Barthes a Eco, sarebbe, in quanto atto non passivo e di mera ricezione, bensì attivo e di costruzione del senso e di spostamento del significato, una forma di creolizzazione. Perciò ogni lettura sarebbe di per sé creola, in quanto gesto di appropriazione e costruzione attiva del senso che nasce non come atto arbitrario di confusione e di mescolamento, bensì come procedura di selezione e di riorganizzazione che emerge da un certo rapporto tra il lettore e il suo mondo, secondo i passaggi già indicati dalla retorica classica (lectio, selectio, electio, intellectio e collectio). Ogni lettura sarebbe dunque una forma di creolizzazione in quanto costituisce un terreno unico di incontro, di interazione e di interpretazione, una fusione originale, come diceva Gadamer, di due orizzonti, che avviene in un intervallo tra due visioni, su una linea permeabile di frontiera, intesa come un'interfaccia tra due griglie di selezione. Piuttosto che una forma di mescolanza e di confusione, l'atto di lettura, così come la creolizzazione per Glissant, è caratterizzata dalla selezione e dal filtraggio, dalla scelta di talune informazioni, immagini o concetti come pertinenti (electio) e dal rigetto di altri elementi ritenuti non-pertinenti. Essa trova quindi una metafora ed un modello nella membrana, intesa come elemento semi-permeabile attraverso la quale avviene questa comunicazione-filtraggio e che garantisce, attraverso questo processo, un arricchimento per l'intero sistema, ossia una intellectio e una collectio che generano il patrimonio di idee e di valori condivisi da una comunità, il "bene comune". Partendo da questa lettura originale della teoria della creolizzazione, Yves Citton definisce una ontologia della soggettività come membrana, da cui deduce una prospettiva politica della moltitudine che contiene elementi estremamente interessanti. Per quanto riguarda la questione, estremamente attuale, delle frontiere e della ricerca di nuove forme politiche nella loro definizione, egli propone di sostituire alla falsa e pericolosa alternativa tra purezza e mescolamento, una difficile riflessione su nuovi "criteri di selezione". Egli afferma che «toute réflexion sérieuse sur la créolisation doit moins se fixer pour but d'abattre des frontières que de les redéfinir, avec pour enjeu principal de sélectionner de critères de sélection plus satisfaisantes (et moins criminels) que ceux actuellement en place» (p. 209). Una delle caratteristiche fondamentali, secondo 
Glissant, della creolizzazione è proprio quella "produzione di imprevedibile", per cui tutti i processi di spostamento, piacevoli o dolorosi, spinti da volontà e desiderio o da necessità, contribuiscono a generare la funzione sociale fondamentale del rinnovamento, sollecitano e liberano quella "potenza della variazione" sulla quale si fonda la dinamica dell'invenzione. «En tant que procédure d'expérimentation», afferma Citton, «de génération de variations et d'émergence du nouveau, la créolisation doit être libre de remettre en question toutes les frontières et de reconfigurer toutes les catégories préexistantes» (p. 209). Si rendono perciò necessarie la prudenza etica e la precauzione, nel senso del Caute! spinoziano, nonché uno sforzo di previdenza e di disponibilità nei confronti dell'imprevedibile, insomma, un'etica ed una politica del filtraggio. Non si tratta, sul piano ontologico, di concepire un essere, individuale e collettivo, circondato da una membrana, bensì un essere la cui essenza stessa consiste in una membrana, in una griglia di selezione e di filtraggio nei confronti del "Toutmonde" di cui siamo parte attiva e costituente. Su questa ontologia può quindi costruirsi una politica rivolta al quotidiano e ai momenti di scelta, di selezione e di opposizione che tramano, come diceva Foucault, le micropolitiche che compongono il corpo collettivo della moltitudine di cui siamo parte e che possono per giunta influenzarlo e modificarlo. Le pratiche di affinamento, a livello del vissuto quotidiano, di queste "politiques membraniques" fondate sulla scelta, l'elezione, l'opposizione ed il rifiuto saranno il banco di prova, negli anni a venire, della messa in atto della creolizzazione planetaria.

Conclude questa stimolante sezione della rivista un articolo di Madison Smart Bell, intitolato Kreyol pale, kreyol konprann (pp. 213-221) che restituisce la creolizzazione al contesto etico, razziale e linguistico generatosi dalla rivoluzione haitiana. 